\title{
Final Technical Report for DOE Grant DE-FG03-97ER54438
}

PI: P. M. Bellan

Applied Physics, Caltech, Pasadena CA 91125

December 21, 2004 


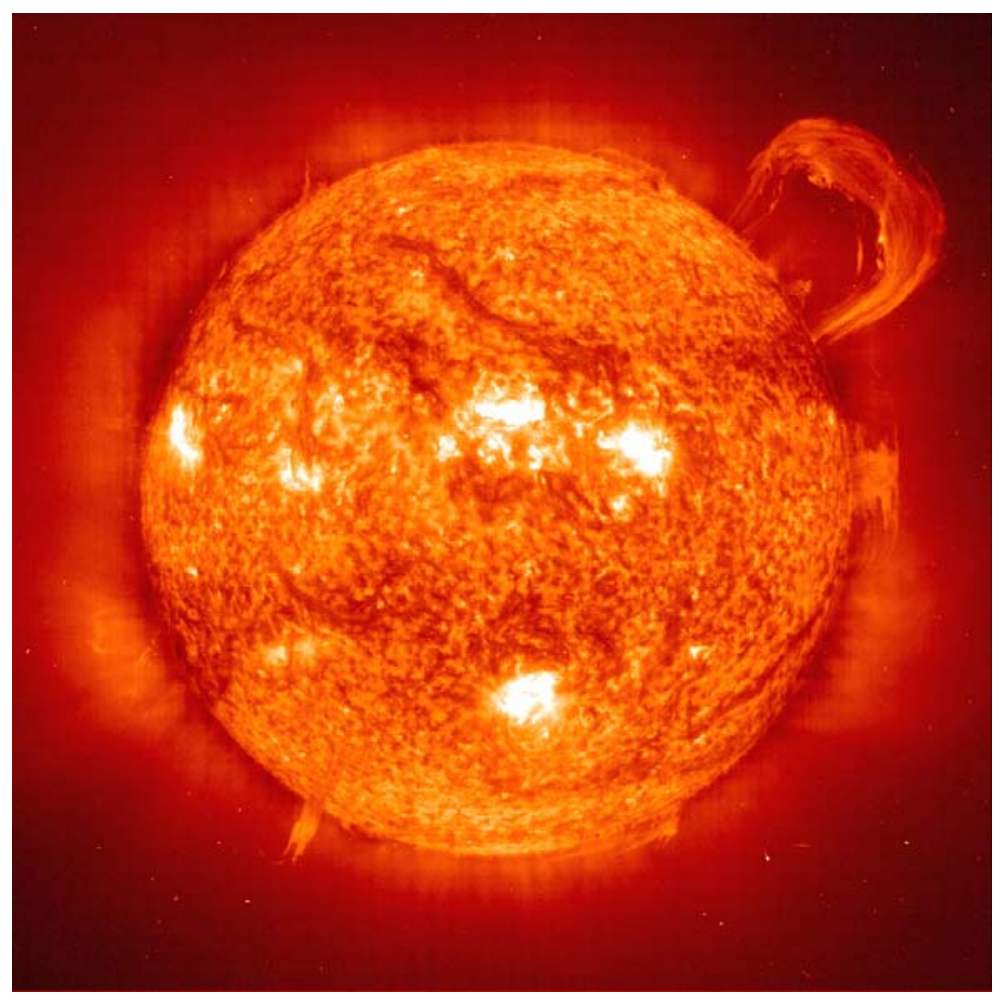

Figure 1: Skylab photo of solar prominence.

\section{Context}

Solar coronal loops (also known as prominences) are arched structures protruding from the solar surface. They are known to consist of plasma-filled magnetic flux tubes and occur at many different scales. Figure 1 shows a dramatic erupting prominence photographed by the NASA Skylab mission.

The research program described in this Final Technical Report had the goal of producing laboratory simulations of solar prominences, i.e., laboratory simulations of what is shown in Fig. 1. These simulations were achieved by using spheromak technology [1] as the basis for a unique magnetized plasma gun which operated in a dimensionless parameter regime similar to solar prominences and which had boundary conditions similar to solar prominences. Being in the same dimensionless parameter regime and having similar boundary conditions means that the simulated prominence is governed by the same magnetohydrodynamic (MHD) physics as an actual prominence. In order to simulate solar conditions a low $\beta$ plasma-filled arched flux tube with significant field-aligned current is created and then allowed to evolve according to its own self-consistent MHD dynamics. This takes place in a large volume which simulates the semi-infinite 
space above the solar surface. MHD physics causes the simulated prominence to pinch, twist, writhe, and bulge just like an actual solar prominence.

The physics governing spheromaks and prominences is, in principle, exceedingly complex because it involves a large number of coupled non-linear partial differential equations incorporating many variables and describing non-trivial three-dimensional topology. However, this complexity is neatly side-stepped by the Woltjer-Taylor relaxation [2, 3, 4] model wherein it is argued that on the relevant time and space scales, magnetic energy is dissipated by instabilities whereas magnetic helicity $[5,6]$ is conserved. Thus, instabilities cause the plasma to seek its minimum magnetic energy, helicity-conserving state. This "relaxed state" is characterized by the simple force-free equation

$$
\nabla \times \mathbf{B}=\alpha \mathbf{B}
$$

where $\alpha$ is a spatially uniform parameter and assumes the smallest value consistent with imposed magnetic boundary conditions. While definitely an oversimplification, Taylor relaxation provides much useful insight and is an excellent way to begin analysis of these complicated structures.

One of the first deviations from ideal Taylor relaxation involves allowing $\alpha$ to be spatially dependent so $\alpha=\alpha(\mathbf{r})$. The divergence of Eq.(1) shows that $\alpha(\mathbf{r})$ must be constant on a field line and so these partially relaxed force-free states can only have $\alpha$ gradients perpendicular to magnetic field lines. $\alpha$ then plays a role in MHD analogous [1] to that played by temperature in thermodynamics, namely $\alpha$ gradients drive helicity flux across field lines [7] just as temperature gradients drive heat flux. A fully relaxed state (i.e., uniform $\alpha$ state) is thus analogous to an isolated thermodynamic system in thermodynamic equilibrium, i.e., a state with uniform temperature.

Solar prominences and spheromaks are moderately well described by Eq.(1) if one allows non-uniform $\alpha(\mathbf{r})$. Solar prominences can be considered as solutions to Eq.(1) with magnetic boundary conditions prescribed on the solar surface, i.e., a solar prominence is an arched flux tube satisfying Eq.(1). If $\alpha$ is zero, the magnetic field is a potential field, just like the field spanning the north and south poles of a horseshoe magnet, whereas if $\alpha$ is finite, then there exists a field-aligned current.

\section{Experimental method}

Figure 2 sketches our basic experimental configuration and its operational sequence and Fig. 3 shows the actual layout of the magnetized plasma gun. A horseshoe electromagnet with $8 \mathrm{~cm}$ separation between poles produces an initial quasi-stationary arch-shaped potential magnetic field, i.e., a situation corresponding to $\alpha=0$. The horseshoe magnet is mounted on the wall of a $1.4 \mathrm{~m}$

diameter, $2 \mathrm{~m}$ long vacuum chamber and is oriented so that the arched magnetic field protrudes into the vacuum chamber interior. 

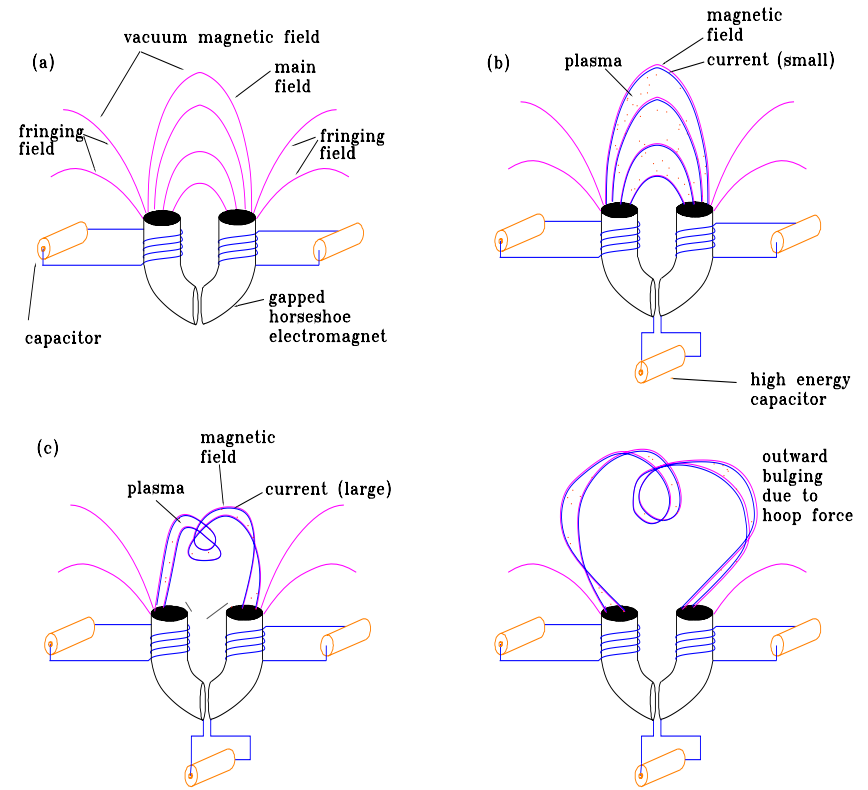

Figure 2: Experimental method: (a) initial vacuum field from gapped horseshoe magnet, (b) small plasma current follows initial arched field lines, (c) large plasma curent causes field lines to pinch and twist, (d) very large current causes arched field lines to bulge outwards.

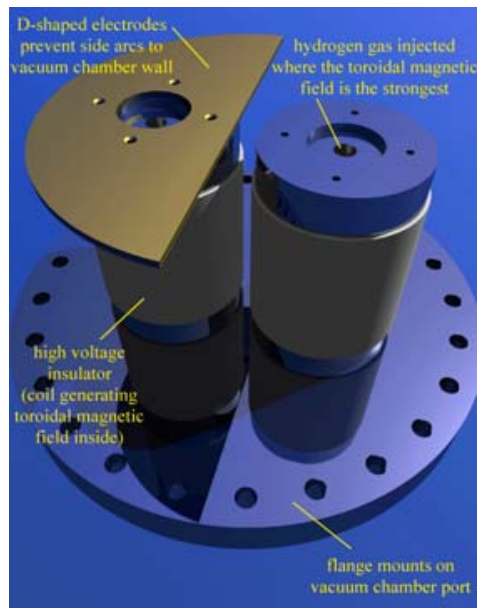

Figure 3: Layout of actual 'solar prominence gun' which creates simulation of prominences. 
The two magnet poles are electrically insulated from each other by a gap in the horseshoe structure [cf. Fig.1(a)]. Hydrogen gas is puffed into the region between the magnet poles and then a high energy capacitor is discharged across the magnet poles [cf. Fig.1(b)]. The applied capacitor potential of several kilovolts quickly breaks down the neutral gas to form nearly fully ionized plasma. The discharging capacitor then proceeds to ramp up an electric current which is ultimately 30 to 80 kiloamps depending on the initial charge voltage of the capacitor.

Initially, when this current is small it simply follows the arched potential field lines [cf. Fig. 2(b)]. However, as the current increases, its associated self magnetic field pinches the current channel so that the channel develops a uniform cross section. Kinking due to interaction between the self-field and the current channel causes the current channel to writhe [cf. Fig. 2(c)] so that the current channel axis becomes helical. Finally, for very large currents, the upward current on one leg of the prominence and the downward current on the other leg mutually repel, leading to an increase of the current channel major radius [cf. Fig. 2(d)]. It should be noted that this driving of electric current along a flux tube corresponds to injecting magnetic helicity into the flux tube.

By appropriate choice of external circuit capacitance and inductance, the rise time of the current is arranged to be slow compared to the characteristic Alfvén time, yet fast compared to the resistive diffusion time. The former constraint ensures that the system is in quasi-equilibrium while the latter ensures that magnetic flux is frozen into the plasma except perhaps at thin, localized reconnection layers.

\section{$3 \quad$ Experimental results}

Increasing the current corresponds to increasing $\alpha$ and thus changing the equilibrium in ways easily understood in terms of MHD. Specifically, as the current increases, it is observed that:

1. The simulated prominence pinches down to have a nearly uniform crosssection along its length. This is in contrast to the very low current $\alpha \simeq$ 0 situation where the arched flux tube has a non-uniform cross-section characteristic of potential magnetic fields.

2. The axis of the simulated prominence writhes such that, when viewed from above, its projection onto the ground plane has an $S$-shape (sigmoid).

3. The simulated prominence is locally twisted.

4. The simulated prominence may subdivide into braided filaments which are twisted and wrapped around each other.

5. The sense of twist, i.e. the chirality, reverses when the electromagnet polarity is reversed. 
Typical photos of the experimental evolution are shown in Fig. 4. The experiment evolves through these configurations in about $10 \mu$ s and the simulated prominence undergoes significant changes on a characteristic time scale of about $0.5-1 \mu \mathrm{s}$. Quantitative studies are undertaken by systematic variation of control parameters such as capacitor charge voltage and electromagnet current. It takes about two minutes to recharge the capacitor banks and have a fresh plasma shot. The plasma is quite reproducible so that detailed studies can be made by firing a large number of plasma shots that are either identical or differ in some chosen controlled fashion.

Fig. 5 shows an image where the plasma gun has been rotated $45^{0}$ to give an oblique view. The simulated prominence is at a late stage where it is quite twisted and its major radius is bulging. The semi-circular extended electrodes are clearly visible.

The setup sketched in Fig.6 has been used [8] to show that the rate of eruption can be controlled by an external, separately generated, over-arching potential strapping field (green lines in sketch) which inhibit eruption altogether if sufficiently strong. The magnet poles are at the two circular depressions in the electrodes (see inset in upper right of Fig.6).
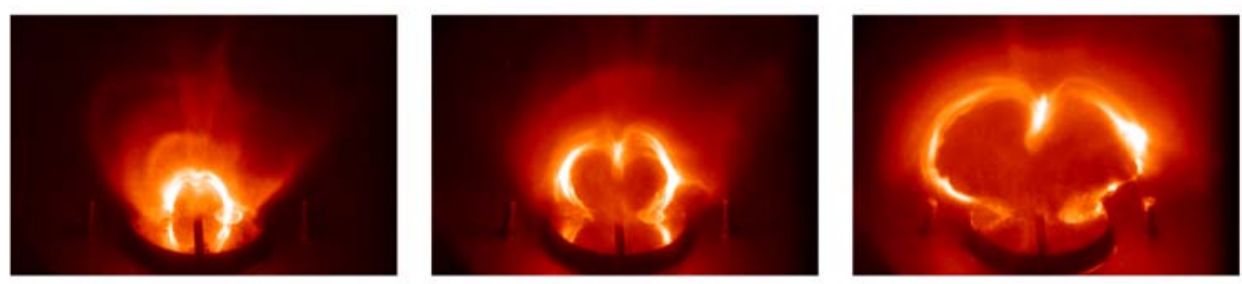

Figure 4: Photos showing typical experimentally observed evolution of simulated prominence. Times are 3.5, 4.5, and $5.5 \mu \mathrm{s}$; footpoint separation is 8 $\mathrm{cm}$. Extended electrodes are visible as semi-circular plates. Stereographic photos show that what is being observed is the projection of a corkscrew (helical) pattern.

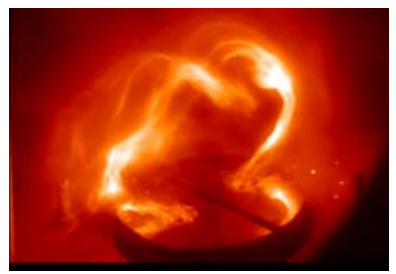

Figure 5: Image at oblique angle. 


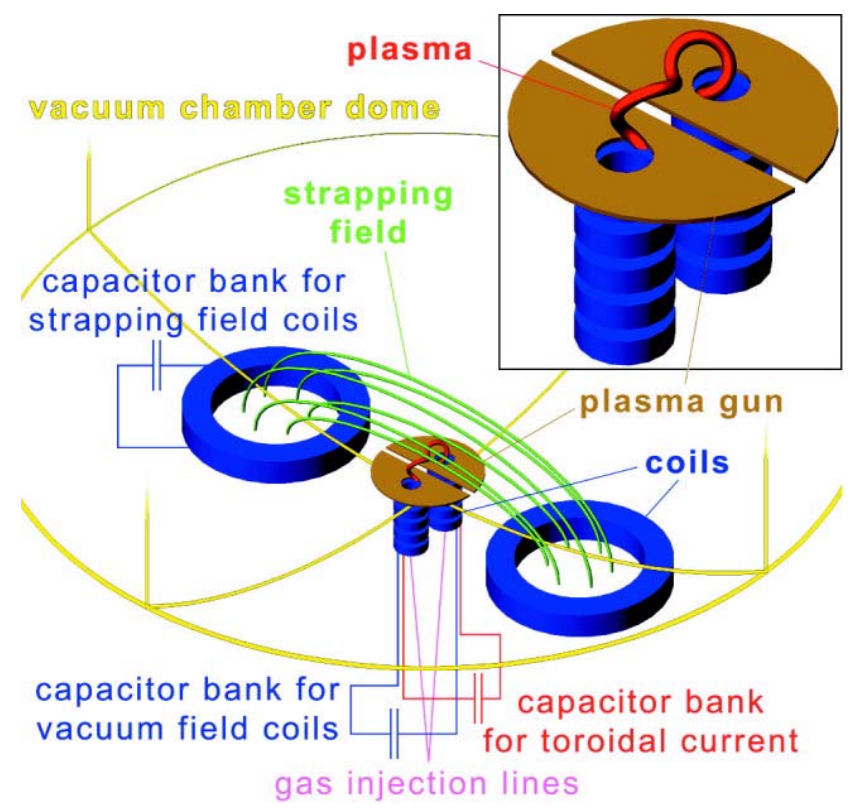

Figure 6: Strapping field setup [8].

\section{Model for MHD flow acceleration, stagnation, heating, finite beta}

Because the experiments are reproducible, they can be used to verify or challenge existing models and also to motivate and guide new models. A new model has been developed $[9,10]$ which goes beyond the standard Taylor relaxation argument to characterize dynamical behavior. This model was motivated by a combination of factors, in particular (i) remarks [11, 12] and publications $[13,14]$ by several solar physicists stating that it is a mystery why prominences always tend to have a nearly axially uniform cross-section, (ii) observations that the lab prominences also have axially uniform cross-sections, (iii) an observed strong sensitivity of the lab prominences to how gas is injected, and (iv) previous investigations by the author on MHD arcjet physics [15]. The new model shows that previously ignored issues of plasma compressibility, finite beta, and flows are all essential to the establishment of equilibrium. In particular, the new model predicts $[9,10]$ that when a current is made to flow along an initially axially non-uniform flux tube (i) unbalanced MHD forces ingest plasma from the footpoints and drive this ingested plasma into the flux tube, (ii) stagnation of the flows at the axial midpoint of the flux tube heats the ingested plasma, and (iii) accumulation and concentration of magnetic flux frozen into the stagnating flows causes the flux tube to tend towards axial uniformity. 


\section{3D Numerical MHD Calculations}

M. Tokman, a Caltech Applied Math PhD student for whom P. M. Bellan was co-advisor, developed a powerful, time-resolved 3D MHD numerical model for coronal mass ejections. The model was motivated in part by the laboratory experiments and, in particular, invoked boundary conditions developed from consideration of the boundary conditions in the laboratory experiment. The results of a typical 3D numerical MHD calculation are shown in Fig.7. This work was published in the Astrophysical Journal [16].
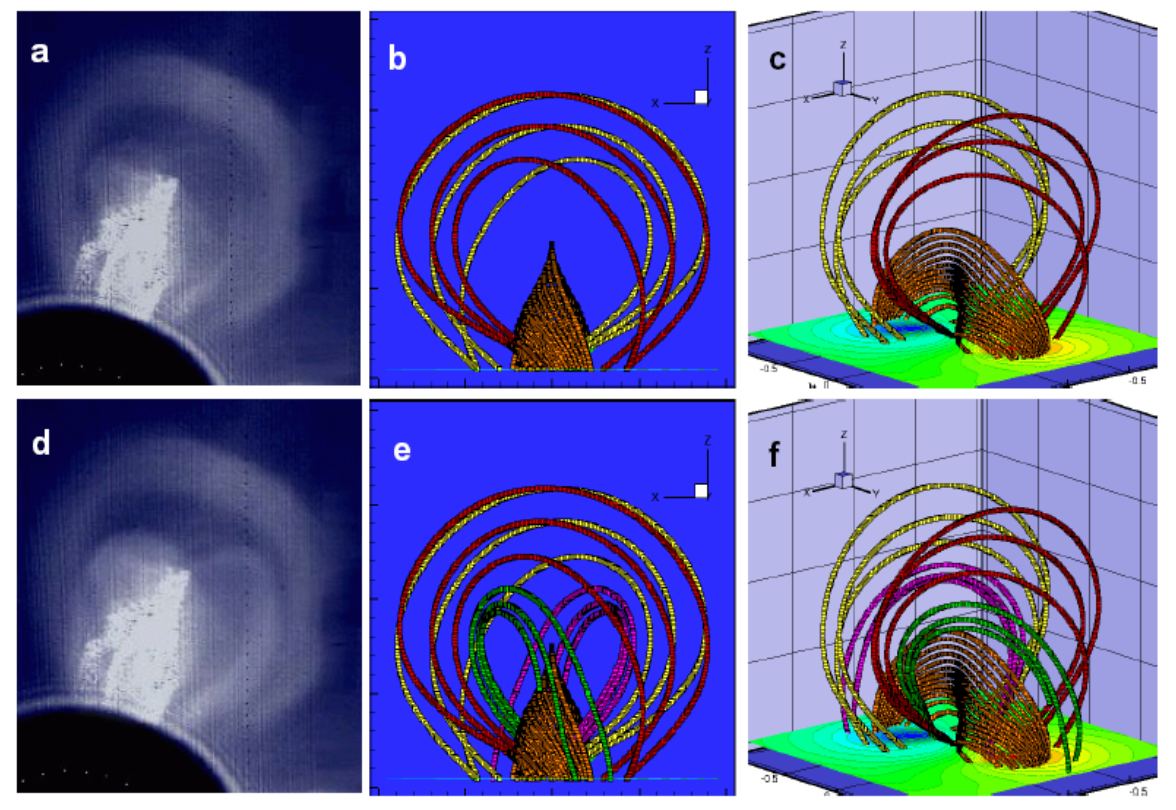

Figure 7: (a) and (d) show photos of actual solar corona mass ejections. (b), (c), (e), and (f) show M. Tokman's 3D numerical MHD calculations [16] indicating morphology similar to (a) and (d).

\section{Impact, outreach, and human resources}

Publications: Five refereed papers on the solar prominence experiment and related issues have been published during the three year period of this grant $[8,9,16,17,18]$.

Thesis award: M. Tokman's thesis won the 2002 Outstanding Dissertation award from APS/DPP.

Invited Talks: P. M. Bellan gave invited talks in 2001 on the solar prominence simulation experiments to audiences at the Lockheed Martin Solar and Astro- 
physics Laboratory (TRACE team), at Swarthmore College, and at the International School/Symposium for Space Plasma Simulations (ISSS-6, Garching). J. F. Hansen gave an invited talk on the solar prominence simulation experiments at the 2001 conference "Black Holes: Theory Confronts Reality, Three Years Later" held at the Institute of Theoretical Physics, UCSB. P. M. Bellan gave invited talks in 2002 at the University of Michigan, at the APS/DPP meeting in Orlando [19], and at the Magnetic Reconnection conference in Hakone, Japan; these talks were on the new model explaining axial uniformity of prominences. M. Tokman gave invited talks at NRL, LANL, and HAO in 2001/2002 and also gave an invited talk [20] at the 2002 APS/DPP meeting in Orlando.

Television Program: The Australian science television program "Quantum" featured our solar prominence experiments in a 2001 broadcast. A TV journalist and cameraman spent a day at Caltech interviewing P. M. Bellan and videotaping experimental operations by Hansen and Bellan.

Distinguished Lecturer: P. M. Bellan served as an APS-DPP Distinguished Lecturer for 2001 and put together a general audience talk consisting of an $\mathrm{MHD}$ /solar plasma tutorial followed by a description of the Caltech solar prominence simulation experiments. He gave this talk during 2001 to physics departments at Utah State University, Florida State University, California State University Sacramento, Montana State University, Loyola Marymount University, the University of Calgary, and the University of Lethbridge (the Distinguished Lecturer Program paid for all the travel except to the Canadian institutions which paid individually).

Popular Article: P. M. Bellan wrote an article on the solar prominence simulation experiments for American Scientist [21]. The Solar Physics Division of the American Astronomical Society gave P. M. Bellan its 2001 Award for Popular Writing on Solar Physics for this article.

Human Resources: J. F. Hansen who did his PhD thesis research on the solar prominence simulations, graduated in 2001 and joined B. Remington's laboratory astrophysics group at the Lawrence Livermore National Lab. M. Tokman, who did her thesis on a 3D numerical model of coronal mass ejections, became a visiting assistant professor at the UC Berkeley Mathematics Department.

\section{References}

[1] P. M. Bellan, "Spheromaks" (Imperial College Press, 2000).

[2] L. Woltjer, "A theorem on force-free magnetic fields", Proc. Nat. Acad. Sci. (USA), 44,489 (1958).

[3] J. B. Taylor, "Relaxation of Toroidal Plasma and Generation of Reverse Magnetic Fields", Phys. Rev. Lett. 33,1139 (1974).

[4] J. B. Taylor, "Relaxation and magnetic reconnection in plasmas", Rev. Modern Phys. 58, 741 (1986). 
[5] H. K. Moffatt, "Magnetic Field Generation in Electrically Conducting Fluids" (Cambridge Univ. Press, Cambridge, England, 1978).

[6] M. A. Berger and G. B. Field, "The topological properties of magnetic helicity", J. Fluid Mech. 147, 133 (1984).

[7] J. C. Fernandez, B. L. Wright, G. J. Marklin, D. A. Platts, and T. R. Jarboe, "The $m=1$ helicity source spheromak experiment", Phys. Fluids B 1,1254 (1989).

[8] J. F. Hansen and P. M. Bellan, "Experimental demonstration of how strapping fields can inhibit solar prominence eruptions", Astrophys. J. 563, L183 (2001).

[9] P. M. Bellan, "Why helicity injection causes coronal flux tubes to develop an axially invariant cross-section", Advances in Space Physics 32, 1923 (2003),

[10] P. M. Bellan, "Why current-carrying magnetic flux tubes gobble up plasma and become thin as a result", Phys. Plasmas 10 Pt 2, 1999 (2003).

[11] U. Feldman, private communication.

[12] B. De Pontieu, private communication.

[13] J. A. Klimchuk, Solar Physics 193, 53 (2000).

[14] J. A. Watko and J. A. Klimchuk, Solar Physics 193, 77 (2000).

[15] P. M. Bellan, Vorticity model of flow driven by purely poloidal currents, Phys. Rev. Letters 69, 3515 (1992).

[16] M. Tokman and P. M. Bellan, "Three-dimensional model of the structure and evolution of coronal mass ejections", Astrophys. J. 567, 1202 (2002).

[17] P. M. Bellan, J. Yee, and J. F. Hansen, "Spheromaks, solar prominences, and Alfvén instability of current sheets", Earth, Planets, and Space 53, 495 (2001).

[18] P. M. Bellan, "Alfvén wave instability of current sheets in force-free plasmas: Comparison to ion acoustic instability", Advances Space Res. 28, 729 (2001).

[19] P. M. Bellan, "Why current-carrying magnetic flux tubes gobble up plasma and become thin as a result", Bull. Am. Phys. Soc. 47, 21 (2002).

[20] M. Tokman, "Three dimensional model of the magnetic structure and dynamics of coronal plasma", Bull. Am. Phys. Soc. 47, 322 (2002).

[21] P. M. Bellan, "Simulating solar prominences in the laboratory", Am. Scientist 88, 136 (2000). 